\title{
La Gestión de la colección en las bibliotecas universitarias españolas. Planes y normativas: la selección
}

\author{
José Luis Herrera-Morillas* \\ * Universidad de Extremadura. Departamento de Información y Comunicación. \\ Correo-e: jlhermor@unex.es
}

Recibido: 21-05-2012; 2a version: 10-07-2012; Aceptado: 12-07-2012.

Cómo citar este artículo/Citation: Herrera-Morillas, J. L. (2013). La Gestión de la colección en las bibliotecas universitarias españolas. Planes y normativas: la selección. Revista Española de Documentación Científica, 36(4):e021. doi: http://dx.doi. org/10.3989/redc. 2013.4.986

Resumen: En este trabajo se estudian 42 textos que regulan la gestión de la colección en las bibliotecas universitarias españolas. Se describe de manera general la gestión de la colección según se recoge en los documentos, prestando especial atención a la estructura y contenidos de los documentos; a la finalidad de los planes de gestión de la colección; y a su concepto y objetivos. En segundo lugar, se profundiza en cómo se plantea el procedimiento de la selección, como una primera parte de esta investigación que será completada en trabajos futuros que abordarán otros aspectos de la gestión.

Palabras clave: Gestión de la colección; selección; bibliotecas universitarias; España; normativas; planes.

Collection management in Spanish university libraries. Plans and regulations: Selection

Abstract: This work studies 42 texts that regulate collection management in Spanish university libraries. Collection management is described in a general way, as it is in the documents. The focus is on the structure and contents of the documents, the aim of the collection management plans, and their concept and objectives. Subsequently, this study details how the selection procedure is presented as the first part of the investigation that will be completed in future works that will deal with other aspects of management.

Keywords: Collection management; selection; university libraries; Spain; regulations; plans.

Copyright: (c) 2013 CSIC. Este es un artículo de acceso abierto distribuido bajo los términos de la licencia Creative Commons Attribution-Non Commercial (by-nc) Spain 3.0. 


\section{INTRODUCCIÓN}

El propósito de este trabajo ha consistido en localizar, recopilar y estudiar los documentos elaborados por las bibliotecas universitarias españolas en los que se plasma la política de gestión de la colección. En primer lugar, se ha querido dar una visión de conjunto de esta política según se desprende de los documentos localizados; y en segundo lugar, se ha analizado con más detalle uno de los aspectos de esta política: la selección.

Conocer y establecer un estado de la cuestión sobre la gestión de la colección es oportuno debido a que las bibliotecas en general, y las universitarias especialmente deben prestar atención a la gestión de la colección, es recomendable redactar un documento que plasme la política de gestión de la colección debido a que:

"las bibliotecas universitarias afrontan numerosos retos presentados por las innovaciones y la evolución de los sistemas de enseñanza, aprendizaje e investigación; las modificaciones en las políticas educativas; los vertiginosos avances de las tecnologías de la información y las telecomunicaciones, y las transformaciones en el mercado editorial y en el modelo de producción y comunicación científica y académica" (Política de gestión de las colecciones, Biblioteca de la Universidad Complutense).

Por ejemplo, el crecimiento de los recursos en formato electrónico o la posibilidad, cada vez mayor, del acceso abierto, hacen necesario y útil disponer de un programa de gestión y desarrollo de la colección que oriente al personal responsable de gestionar la colección, y que "también sirva para informar a la comunidad universitaria sobre la misión, los objetivos y los procedimientos vinculados a las colecciones" (Plan de gestión y desarrollo de la colección, Biblioteca de la Universidad Politécnica de Cataluña).

Además, estos retos exigen una puesta al día constante y una redefinición frecuente de los objetivos, las estrategias y los procedimientos de trabajo, siempre con la pretensión de satisfacer las necesidades actuales de los usuarios (Política de gestión de las colecciones, Biblioteca de la Universidad Complutense).

\subsection{Desarrollo y gestión de la colección}

Existe cierta ambigüedad respecto al uso de las expresiones desarrollo o gestión, aplicadas a la colección de materiales de una biblioteca (ver Fuentes, 2010; Solimine, 2010; Aguado, 2011).

Así, hay autores que entienden el desarrollo de la colección como el establecimiento de los principios y objetivos generales en torno a los cuales se va a ir construyendo la colección. De tal modo que la gestión queda dentro del desarrollo:

"El desarrollo de la colección es el proceso de planificar, construir y mantener los recursos de información de una biblioteca de modo que sea eficiente en su coste y relevante para sus usuarios" (Gabriel, 1995)

Para otros autores la gestión abarca los procesos más concretos de la preparación de la colección, fundamentalmente: selección, adquisición, organización, presupuesto, colocación, etc.

También, hay autores para los que la diferencia entre desarrollo y gestión de la colección es prácticamente inexistente:

"Hablamos de gestión o desarrollo de la colección para referirnos a todo lo relacionado con su planificación, formación, evaluación y mantenimiento. Abarca varias actividades como la determinación de los criterios de selección, evaluación de necesidades, proceso de adquisición, estudios del uso de la colección, evaluación, cooperación para compartir recursos, conservación y expurgo" (Gómez Hernández, 2002).

En los años 50 del siglo $X X$ se fechan los primeros estudios de cierta entidad, relacionados con las colecciones, centrados sobre todo en los procesos de selección y adquisición. En la década siguiente, en Estados Unidos se empieza a aplicar una orientación más ambiciosa bajo la expresión "desarrollo de colecciones". Como explica Aguado (2011), este nuevo enfoque supone contar con:

"la determinación previa de las características de la unidad de información, la postulación de objetivos en concordancia con las aspiraciones de la comunidad de usuarios a la que se pretende servir, la evaluación de la colección a intervalos regulares, para descartar o relegar los materiales de poco o ningún uso y detectar necesidades de información no satisfechas, y, sobre todo, la búsqueda de procedimientos para satisfacerlas, tanto con fondos documentales propios como mediante convenios para uso compartido".

En los años 80 se comienza a utilizar gestión de la colección, a modo de concepto-paraguas que englobaría al desarrollo de la colección. Incluyendo "el desarrollo de la colección y todas las decisiones acerca del expurgo, la cancelación de suscripciones, el almacenamiento y la preservación" (Fuentes, 2010).

Hoy en día está más extendida la expresión "gestión de colecciones", con un planteamiento amplio que incluye junto a los procesos clásicos (selección, adquisición, etc.) otros como los planteamientos previos, la conservación o el mantenimiento.

Dentro de los elementos y componentes de la gestión de la colección es fundamental abordar la metodología de desarrollo y gestión de las colecciones; para Solimine (2010) se compone de:

- La definición de la fisionomía documental de una biblioteca.

- La identificación de las fuentes de información a través de las cuales controlar la producción editorial corriente. 
- La selección atenta y meditada de los documentos a adquirir.

- El trabajo de tratamiento y medición de los documentos introducidos en la colección.

- El control constante del uso.

- La continua renovación de la oferta, por una parte a través del incremento de las colecciones y por otra, a través de la eliminación de todo lo que no sea funcional a la satisfacción de la demanda.

Para Evans (1995) es un proceso continuo compuesto de 6 elementos definibles:

- Análisis de la comunidad (usuarios).

- Política de la colección (normas y estándares).

- Selección.

- Adquisición.

- Expurgo.

- Evaluación.

La ALA en su Guide for written collection policy estatements (1996) establece como elementos que debe abarcar la política de la colección:

- Introducción (contexto, misión, objetivos, etc.).

- Aspectos generales para la gestión y desarrollo de la colección (tipos de publicaciones, materias, formatos, etc.).

- Análisis detallado de las materias de la colección.

- Análisis detallado de las colecciones especiales.

- Niveles de la colección.

- Códigos de lengua.

La IFLA inspirándose en el documento de la ALA redacta sus Directrices para una política de desarrollo de las colecciones sobre la base del modelo Conspectus (2001), que establecen como elementos a tener en cuenta los siguientes:

- Introducción (misión de la biblioteca, público al que va dirigido, etc.)

- Principios generales (características que determinan la orientación del desarrollo de la colección).

- Principios particulares (para reflejar la biblioteca en particular).

- Perfiles de materias (se recomienda los informes Conspectus).

- Métodos de evaluación de la colección (medidas cualitativas, cuantitativas e indicadores de profundidad de la colección).

- Códigos de lengua (RLG).

- Códigos de lengua (WLN).
Otra expresión muy utilizada en relación con la gestión de la colección es la de Políticas de la colección. Responde a la necesidad, sobre todo en las grandes bibliotecas, de no improvisar en todo lo relacionado con el crecimiento de la colección, de lo contrario el presupuesto no se aplicará correctamente ni se satisfarán todas las necesidades de manera correcta y profesional.

Las fases para trazar la política de las colecciones de una biblioteca son variadas e implican a diferentes personas. Futas (1995) habla en secuencia cronológica de las siguientes:

Se fija la tarea del grupo de trabajo encargado de la planificación.

- Se recogen los datos e informaciones sobre los cuales tomar correctamente las decisiones.

- Se elabora un documento de policy statement.

- Se aplican los contenidos de dicho documento programático.

El documento programático, también denominado la carta de las colecciones, es de gran ayuda. En él se exponen, por ejemplo: los parámetros de referencia preseleccionados; las prioridades; el nivel de profundidad para las diferentes áreas temáticas; los criterios que adoptan para la selección del material, etc. Para Solimine (2010) la carta:

"explicita y comunica (a todo el staff de la biblioteca, a los usuarios, a los administradores y financiadores de la estructura, a las demás bibliotecas con las cuales se tienen relaciones de cooperación) los objetivos de la política de las colecciones, vinculándolos a los fines generales de la estructura".

El esquema que propone para la carta consta de:

- Fines de la biblioteca y de sus colecciones.

- Perfil de la comunidad.

- Estrategias de servicio.

- Características de las colecciones.

- Cooperación.

- Responsabilidades de decisión y modalidades operativas.

Gardner (1981) habla del estatuto de las colecciones como ayuda e instrumento de trabajo al que asigna estas funciones:

- Incitar a los responsables a tener siempre presentes los fines de la biblioteca y a medirse con ellos, así como a identificar las necesidades de los usuarios y a establecer un orden de prioridades en el reparto de los recursos.

- Considerar orgánicamente todos los componentes de la comunidad o de la institución que se sirve. 
- Establecer un estándar para la selección o el expurgo del material.

- Informar a los usuarios, los administradores y las otras bibliotecas de los sectores disciplinarios de la colección, a fin de facilitar las relaciones de cooperación.

- Limitar la discreción personal en las operaciones de selección, dando reglas uniformes y reconduciendo las decisiones individuales en el seno de una política común.

- Suministrar un instrumento de formación y orientación al personal neo-empleado.

- Asegurar la continuidad y facilitar la transición entre los bibliotecarios que se alternarán en el trabajo de selección.

- Suministrar un parámetro de referencia para la actividad de valoración y autovaloración.

- Favorecer la afirmación de una concepción de la biblioteca como agenda productora y distribuidora de servicios.

- Favorecer las relaciones con el público, haciendo más fácilmente comprensibles las razones de las decisiones de inclusión o exclusión.

- Suministrar informaciones útiles a la definición de las políticas de balance.

Tras definir las características esenciales y tamaño de una colección (normalmente a través de la carta de las colecciones) se aconseja llevar a cabo una política de desarrollo (Solimine, 2010) que responda a ese trabajo previo y que plasme cada año los objetivos definidos en la carta de las colecciones y el uso de los recursos financieros disponibles. Los rasgos de la política de las adquisiciones se precisan en un plan de desarrollo de las colecciones, que -a grandes rasgos- el citado autor define como un reparto del presupuesto anual. Pero, que en otros casos más complejos puede llegar a ser un documento más elaborado que tenga en cuenta:

"los objetivos y los resultados de los planes de desarrollo de los años anteriores y que a veces puede incluso introducir algún elemento de innovación respecto a la carta de las colecciones en espera de una revisión (por ejemplo, por la creación de una nueva titulación en el caso de una biblioteca universitaria...)".

De esto modo, los resultados de la reflexión previa y de las decisiones finales serán:

- La presentación de los objetivos prioritarios para el año al cual se refiere el plan, en función de lo previsto por la carta de la colecciones y de los resultados del plan de desarrollo del año precedente.

- La traducción de estos objetivos en cantidad de documentos que nos proponemos adquirir por cada sector.
- El reparto del presupuesto para los diferentes sectores.

- La indicación de las sumas reservadas a la satisfacción de las demandas de los usuarios.

- La actitud que se piensa tomar respecto a cuestiones particulares, como la adquisición de varios ejemplares de las ediciones más demandadas.

El plan de desarrollo de colecciones tendrá también necesidad, en muchos casos, de ser revisado y modificado durante el año.

\subsection{La selección}

La selección se puede definir como:

"la evaluación y la elección de los materiales que se incorporan a la colección. Es un proceso complejo, que implica una interpretación ajustada de las políticas de la unidad de información, una estimación de la demanda potencial de los usuarios, la disponibilidad de fuentes para conocer la existencia de títulos convenientes, la exploración de las posibilidades de acceso al documento mediante convenios de reciprocidad con otras instituciones" (Aguado, 2011).

Fuentes (2010) explica que en este proceso intervienen unos factores (conocimiento de los materiales, conocimiento de los recursos con que se cuenta, conocimiento de los usuarios); se siguen unas etapas (identificación de los materiales pertinentes, valoración-evaluación, decisión de adquisición, preparación de la orden de adquisición) y unos principios generales (actualidad, interés, público al que va dirigida la selección, neutralidad) ${ }^{1}$.

Los autores también suelen establecer como ayuda en el proceso de selección unos criterios de selección -generales y/o específicos para cada tipo de materiales- (por ejemplo Gómez Hernández, 1997 y 2002; White y Crawford, 1997) y unas fuentes (Aguado, 2011).

La IFLA (2001) en sus Directrices... mantiene que la selección se debe guiar por una "política escrita", de este modo se "reduce el margen de las inclinaciones personales al basar las decisiones sobre la selección en el contexto de los objetivos de la colección".

\section{MATERIAL Y MÉTODO}

Para llevar a cabo este trabajo ha sido necesario, en primer lugar, localizar y recopilar los documentos de las bibliotecas universitarias españolas sobre la gestión de la colección. Para ello se comienza visitando los sitios web de las bibliotecas, y tras comprobar qué bibliotecas tienen accesibles documentos de este tipo y cuáles no, se procede, en segundo lugar, a contactar por correo electrónico con las bibliotecas de las que no se ha encontrado dichos documentos, para preguntarles por su política de gestión de la colección (Anexo I). 
Las bibliotecas universitarias se han localizado a través del directorio Listado alfabético de universidades por comunidades autónomas <http://www. ua.es/es/internet/listado.htm>. Este listado enlaza con las 75 universidades españolas, tanto públicas como privadas. De este listado se han desechado 4 universidades, debido a que por sus características peculiares no parece coherente agruparlas con el resto (Universidad Internacional de Andalucía, Universidad Internacional Menéndez Pelayo, Universidad Oberta de Catalunya, Universidad a Distancia de Madrid). Por lo tanto, la muestra de estudio abarca 71 bibliotecas universitarias (Anexo I).

Los documentos y la información recopilada se han organizado en dos apartados (Anexo I):

1. Bibliotecas que cuentan con planes de gestión de la colección. Por planes de gestión de la colección se entiende los documentos que sirven para:

"establecer los principios generales y las líneas directrices que guiarán en adelante la gestión de la colección [...] los criterios y las directrices básicos para coordinar y llevar a cabo las diferentes actividades de gestión de la colección (Política de gestión de las colecciones, Biblioteca de la Universidad Complutense).

2. Bibliotecas que sólo cuentan con normativas o documentos que abarcan aspectos parciales o concretos relacionados con la gestión de la colección. Por ejemplo: selección, adquisición, expurgo, etc.

Para el estudio de los planes de gestión de la colección se han seguido los siguientes aspectos:

- La denominación empleada en los documentos.

- La estructura y contenidos de los documentos.

- La finalidad de los planes de gestión de la colección.

- El concepto y los objetivos de la gestión de la colección.

Las visitas a los sitios web de las bibliotecas y las consultas por correo electrónico se han realizado entre el 20 de marzo y el 5 de mayo de 2012.

\section{RESULTADOS Y DISCUSIÓN}

De las 71 bibliotecas analizadas, 33 tienen documentos relacionados con la gestión de la colección $(46,47 \%)$. De estas 33 bibliotecas, 10 han elaborado planes de gestión de la colección y 23 bibliotecas, normativas o documentos parciales (Anexo I)

En total se han analizado 43 documentos (10 planes de gestión de la colección y 33 normativas o documentos parciales) (tablas I y III).
Las fechas de aprobación de los planes ( 2 planes no la indican) reflejan que han sido elaborados entre los años 2005 al 2009 (tabla I).

Respecto a las normativas o documentos parciales (tabla II), 13 documentos no indican la fecha. Los 20 restantes oscilan entre los años 1998 al 2011. Los años mejor representados son el 2010 (5 documentos), 2011 (4 documentos), 2002 (3 documentos) y 2004 (2 documentos). Los documentos son recientes lo cual puede mostrar que su elaboración es una práctica también reciente que es previsible se extienda a otras bibliotecas.

\subsection{Planes de gestión de la colección}

\subsubsection{Denominación empleada en los documentos}

De los 10 documentos (tabla I), 8 documentos utilizan la palabra "gestión"; y 3 documentos, además, añaden la palabra "desarrollo". Por otro lado, 2 documentos no emplean ninguna de estas dos expresiones: en un caso sólo se emplea la palabra "colección" y en el otro se enumeran los procesos de "selección, adquisición y descarte".

Esta terminología refleja que la denominación gestión de la colección es la más extendida, si bien no se ha perdido del todo el término desarrollo, muy vinculado al concepto, como hemos mencionado en la introducción.

También, se puede destacar que sólo 4 documentos han elegido el término "plan". El resto se han decidido por "política" (2 documentos), por "programa" (2 documentos), "proceso" (1 documento) y por "criterios" (1 documento). En el empleo de estos términos se aprecia una mayor variedad o dispersión que en los anteriores.

\subsubsection{La estructura y contenidos de los documentos}

Todos los documentos incluyen apartados para la adquisición y para el expurgo (La Autónoma de Barcelona emplea el término retirada). En relación con las adquisición están las donaciones, que la abordan todas las bibliotecas menos una (Alfonso $X$ El Sabio); en la mayoría como uno de los procedimientos o formas de adquisición ( 6 documentos), pero en dos (Sevilla y Barcelona) se tratan en apartados diferenciados.

Todos los documentos menos uno (Barcelona) abordan la selección, con frecuencia en el mismo apartado que la adquisición (6 documentos).

Todos los documentos menos 3 (Barcelona, Málaga, Alfonso X El Sabio) abarcan la evaluación (la Autónoma de Barcelona utiliza un apartado denominado Seguimiento y revisión de los planes de colección que hace referencia más al plan en sí que a la colección). La abordan con más extensión los documentos de la Universidad Politécnica de Ca- 
Tabla I. Denominación empleada en los documentos de planes de gestión de la colección

\begin{tabular}{lll}
\hline Nombre del documento & Universidad (Biblioteca) & Año \\
\hline Criterios para la gestión de la colección & La Rioja & 2006 \\
\hline Plan de gestión de la colección & Málaga & 2007 \\
\hline Plan de gestión y desarrollo de la colección & Politécnica de Cataluña & 2009 \\
\hline Plan de gestión y desarrollo de la colección & Autónoma de Barcelona \\
\hline Plan general de la colección & Complutense de Madrid & 2005 \\
\hline Política de gestión de las colecciones & Alfonso X El Sabio & 2009 \\
\hline Política de selección, adquisición y descarte & Murcia \\
\hline Procedimiento para la gestión de la colección & Carlos III de Madrid \\
\hline Programa general de gestión de la colección & 2007 & 2008 \\
\hline Programa general de gestión y desarrollo de colecciones & Sevilla & 2006 \\
\hline
\end{tabular}

taluña y la Universidad Carlos III, que centran la evaluación en la medición del uso (estableciendo unos indicadores) y partiendo de los criterios definidos para la selección; o el documento de La Rioja que explica la herramienta en File Maker diseñada para obtener información cuantitativa de la colección con el objeto de valorar el uso, la disponibilidad y la adecuación a las necesidades actuales y previsibles de los usuarios.

Los demás textos son más escuetos: el de la Complutense cita la metodología Conspectus y cómo la aplica; el de Murcia está centrado en el análisis de las estadísticas de uso; y el de Sevilla sólo menciona los principios generales que guían la evaluación.

El presupuesto se trata como un apartado destacado en 7 documentos, bajo diferentes denominaciones: "Política presupuestaria" en 5 documentos (La Rioja, Murcia, Politécnica de Cataluña, Carlos III, Sevilla, en este texto aparece como "Política de gestión económica y presupuestaria"); "Aspectos económicos de las colecciones" en 1 documento (Complutense) y "Financiación" en 1 documento (Autónoma de Barcelona). Los documentos más detallados son los de la Universidad de La Rioja que desarrolla aspectos como el órgano responsable, las partidas presupuestarias, los plazos de aprobación, o los criterios de reparto del presupuesto; la Universidad Carlos III que explicita el personal responsable, los porcentajes y las fases por las que pasa el reparto del presupuesto; la Universidad de Sevilla que precisa con detalle los baremos, porcentajes y fórmulas para la asignación del crédito; o la Universidad Complutense que hace una descripción cualitativa de las directrices generales que marcan el reparto del presupuesto.

Con menor extensión lo tratan la Universidad de Murcia: presenta unos rasgos generales de la distribución del presupuesto; o la Autónoma de Bar- celona: se limita a exponer las diferentes partidas presupuestarias para financiar las adquisiciones. En otros textos las alusiones son más reducidas aún (en el de Málaga dentro del apartado Directrices del plan; en el de la Autónoma de Barcelona en Responsabilidad de la selección y aprobación del gasto).

Los proveedores se abordan en 7 documentos como elemento diferenciado del índice. La expresión más utilizada es "Política de proveedores": en 6 documentos (La Rioja, Málaga, Murcia, Politécnica de Cataluña, Complutense, Sevilla). La extensión difiere de unos a otros: en La Rioja se desciende a los detalles que marcan la elección de los proveedores para cada tipo de documento; también se dedica un completo apartado para fijar los aspectos que guían la evaluación de proveedores. La Politécnica de Cataluña establece criterios generales y otros criterios más detallados para la revisión periódica de los proveedores según la tipología documental.

La explicación no es tan detallada en los textos de las otras universidades: la Complutense desarrolla 5 aspectos principales que se tendrán en cuenta a la hora de seleccionar a los proveedores; la de Sevilla informa que la adquisición de los materiales documentales se realiza a través de los proveedores homologados en los concursos de suministros realizados periódicamente y enumera los criterios que se tendrán en cuenta en la evaluación de proveedores; la Universidad de Murcia recoge 5 factores para la selección; y la de Málaga 4 criterios. Los mencionan también, todavía con menos protagonismo, los documentos de la Universidad Alfonso X El Sabio y la Autónoma de Barcelona.

La conservación y/o preservación se recoge en 6 documentos, 3 lo hacen de manera conjunta (Carlos III, Murcia y Politécnica de Cataluña, en esta Universidad formando parte del mantenimiento); en 1 documento (Sevilla) la conservación aparece 
unida a la restauración; en 1 documento (Complutense) únicamente se trata de la preservación; en 1 documento (Málaga) sólo se aborda la conservación.

Los textos de las universidades Carlos III, Sevilla, Murcia y Málaga se limitan a establecer la necesidad de redactar un plan que aborde estos aspectos. Con un mayor desarrollo se incluye en la Universidad Complutense: se basa en los principios de la IFLA (también en la Carlos III) y recoge unas directrices sobre la preservación de documentos en general, otras directrices sobre las colecciones digitales, y unas indicaciones para la seguridad. La Politécnica de Cataluña se detiene en las actuaciones dirigidas a la preservación diferenciando entre las colecciones en soporte físico, fondo antiguo, fondos especiales y materiales digitales.

La frecuencia y generalización de estos temas en los documentos nos indica que son los esenciales en la gestión de la colección, y que con ellos se identifica este concepto en las bibliotecas universitarias españolas.

Estos temas se completan con otros según las bibliotecas, por ejemplo: derechos de autor y de propiedad intelectual (Sevilla, La Rioja, Murcia, Politécnica de Cataluña y Carlos III); desarrollo de la colección (o programas específicos de gestión de la colección) por áreas temáticas (Carlos III, Sevilla); descripción de las colecciones (Politécnica de Cataluña, Autónoma de Barcelona, Murcia); o niveles de la colección (Málaga, Murcia).

Si comparamos la estructura de estos documentos con el esquema propuesto por la ALA (1996) en su Guide... la mayor coincidencia se da en presentar un apartado introductorio con un planteamiento semejante (todas las bibliotecas menos la Alfonso $X$ el Sabio), y en destacar la evaluación. Los únicos documentos que hacen una mención explícita al Conspectus son los de la Biblioteca de la Complutense y de la Politécnica de Cataluña.

\subsubsection{La finalidad de los planes de gestión de la colección}

Los planes se constituyen en algunos casos como un "documento marco": es el caso de la Biblioteca de la Universidad Complutense que redacta su plan con el fin de establecer los principios generales y las líneas directrices que guían la gestión de la colección. Recoge los criterios y las directrices básicos para coordinar y llevar a cabo las diferentes actividades de gestión de la colección. También lo plantean así la Biblioteca Autónoma de Barcelona o la Biblioteca de la Universidad Politécnica de Cataluña:

"El objetivo de este documento es establecer un marco que permita desarrollar una política de gestión de la colección adecuada y coherente en relación con las necesidades de los usuarios de las bibliotecas y los recursos disponibles".
Para otras bibliotecas los planes tienen como finalidad ser una "guía para los responsables de gestionar la colección". Este planteamiento está inspirado en las Directrices...de la IFLA (2001): "una breve guía de cómo llevar a cabo una política de colecciones $[. .$.$] , pretende ser un instrumento, so-$ bre todo, para los colegas [...] que se encargan de las colecciones".

Así lo concibe la Biblioteca de la Universidad Carlos III de Madrid. En la Biblioteca de la Universidad de Sevilla la guía se concreta en establecer las líneas de actuación y fijar los objetivos y metodologías de trabajo en torno al ámbito de la gestión de la colección. En la Biblioteca de la Universidad de Málaga el plan se diseña como una guía de ayuda para formar una colección basada en el estudio de las necesidades informativas reales de los usuarios, de los planes de estudio y líneas de investigación de la comunidad universitaria. Tiene como función establecer objetivos y principios generales, fijar metodologías de trabajo y velar por la adecuación y coherencia de las colecciones.

Para la Biblioteca de la Universidad de Murcia la finalidad es "el estudio de la gestión y desarrollo de las colecciones [...] sea cual sea su fuente de financiación...".

Estos propósitos generales se completan con otros, así en el caso de Sevilla o Murcia se concibe para que sirva de información a la comunidad universitaria sobre la misión, objetivos y procedimiento de la biblioteca en este campo, y para asegurar que la política de gestión de la colección se adecua a los objetivos de la biblioteca; o en el caso de la Biblioteca de la Universidad Carlos III de Madrid debe ser uno de los principales instrumentos de comunicación entre los diferentes estamentos universitarios y la biblioteca, y ayudará al cumplimiento de los objetivos del servicio dentro de la universidad.

Tanto la Biblioteca de la Universidad de Sevilla como la de la Universidad Politécnica de Cataluña matizan que estos documentos se tendrán que complementar y desarrollar en otros programas o líneas que recojan las características individuales de cada una de las bibliotecas en las que se organiza la universidad.

\subsubsection{Concepto y objetivos de la gestión de la colección}

La Biblioteca de la Universidad Complutense concibe la gestión de la colección como un conjunto de actividades de gestión llevadas a cabo con unos criterios y directrices que marcan la política de gestión de la colección:

"qué procedimiento es el más adecuado para adquirir cada tipo de documento, qué proporción entre libros, revistas y otros recursos se ajusta mejor a las necesidades, qué soporte es preferible y optimiza la difusión y la consulta, qué grado de duplicación es el razonable, cómo debería 
equilibrarse el gasto entre la bibliografía básica y el material didáctico para los alumnos, las obras y materiales para la docencia y los recursos para la investigación, qué niveles de alcance son recomendables para cada materia, etc.".

Para la Biblioteca de la Universidad Politécnica de Cataluña la gestión de la colección hace referencia a las actividades relacionadas con la selección y la adquisición de los documentos, el mantenimiento de la colección y el seguimiento de su uso.

En términos muy parecidos la definen las demás bibliotecas: así, para la Autónoma de Barcelona esta gestión incluye los criterios generales de "la selección, la adquisición, la preservación, la evaluación y el descarte de los fondos bibliográficos". Para Málaga la gestión de la colección busca coordinar y facilitar las cuestiones relativas a "la política de selección, adquisición y expurgo de los fondos, control del presupuesto, evaluación del acceso y uso, políticas de preservación y conservación de todo tipo de materiales". En el caso de Murcia "mantener colecciones equilibradas, mediante políticas de selección, adquisición y expurgo de los fondos, corrigiendo las desviaciones que se produzcan". O para la Universidad Carlos III, la gestión de la colección es un objetivo prioritario de la Biblioteca y consiste en:

"coordinar y facilitar las cuestiones relativas a la colección de la Biblioteca en lo referente a política de selección, adquisición y expurgo de los fondos (incluida la política de proveedores) control del presupuesto, evaluación del acceso y uso, políticas de preservación y conservación de todo tipo de materiales".

En algunos documentos se enumeran una serie de objetivos relativos al programa o política de gestión de la colección. Es el caso de las bibliotecas de Sevilla y de la Politécnica de Cataluña que coinciden en estos objetivos:

"-Reflejar la política de la Biblioteca respecto de su colección, teniendo en cuenta las necesidades de sus usuarios y los objetivos institucionales.

-Establecer las líneas de actuación en torno a la Gestión de la Colección: selección, adquisición, evaluación y expurgo.

-Indicar las responsabilidades de cada una de las tareas del proceso.

-Definir las colecciones existentes y planificar su futuro desarrollo.

-Velar por la adecuación, coherencia y equilibrio de las colecciones en relación con los planes de estudio y las líneas de investigación de la Universidad".

Estos planteamientos son muy semejantes a los postulados por la ALA (1996) en su Guide...:

"Un programa de gestión y desarrollo de colección contribuye al logro de aquellos objetivos de los procesos de adquisición y suministro de ma- teriales, los integra en colecciones coherentes, gestiona su crecimiento y mantenimiento y descarte. Tal programa es un elemento que forma parte del plan de la biblioteca para satisfacer las necesidades de acceso a las fuentes de información de la comunidad a la que sirve".

\subsection{Normativas y documentos parciales}

En la tabla II se incluyen las 33 normativas y/o documentos parciales relacionados con la gestión de la colección que hemos localizado.

Se observa en primer lugar que 8 bibliotecas tienen 2 ó más documentos; y que 4 documentos abarcan 2 ó más aspectos (Burgos: adquisición y desarrollo; Cádiz: expurgo y donación; Pontificia de Comillas: mantenimiento y adquisición; Rey Juan Carlos: donación, canje y depósito).

Los 29 documentos restantes están de dedicados a:

- Las adquisiciones y/o suscripciones (12 documentos).

- Las donaciones (8 documentos).

- El expurgo (7 documentos).

- La selección (2 documentos).

\subsection{La selección}

La selección se aborda en los 10 planes de gestión de la colección y en 8 de los demás documentos.

Los elementos más generalizados que abarca el proceso de la selección, tal como se define en estos textos, son:

- Criterios para la selección: 13 documentos (12 documentos recogen criterios generales; 5 específicos; 5 de ambos tipos y 2 para publicaciones periódicas).

- Responsabilidad en la selección: 6 documentos (en la Complutense: "agentes de la selección").

- Fuentes para la selección: 4 documentos.

- Estos aspectos se completan en algunos casos con otros como:

- Principios generales para la selección: 2 documentos.

- Herramientas para la selección en línea: 2 documentos.

- Factores que influyen en la selección: 1 documento.

- Consideraciones generales: 1 documento.

\subsubsection{Los criterios}

Respecto a los criterios generales de selección de los 12 documentos que los incluyen, 9 documentos coinciden en presentar los criterios en forma de 
Tabla II. Normativas y documentos parciales sobre aspectos de gestión de la colección

\begin{tabular}{|c|c|c|}
\hline Universidad (Biblioteca) & Normativa o documento & Año \\
\hline Alcalá & Criterios para la selección de recursos de información & --- \\
\hline Alicante & Normativa de adquisiciones de fondos bibliográficos & --- \\
\hline Alicante & Normativa de suscripciones de publicaciones periódicas y bases de datos & --- \\
\hline Burgos & Instrucciones para la adquisición y el desarrollo de publicaciones & --- \\
\hline Cádiz & Normativa de expurgo y donación & 2010 \\
\hline Cantabria & Servicio de adquisiciones & --- \\
\hline Castilla-La Mancha & Normativa del servicio de adquisiciones & 2004 \\
\hline Católica de Ávila & Normativa de adquisiciones & --- \\
\hline Católica de Ávila & Normativa de expurgo & --- \\
\hline Extremadura & Normativa de admisión de obras por donación & 2007 \\
\hline Extremadura & Manual de procedimiento para las adquisiciones bibliográficas (monografías) & 2010 \\
\hline Huelva & $\begin{array}{l}\text { Normas para la adquisición de material bibliográfico y suscripción a } \\
\text { publicaciones periódicas y bases de datos }\end{array}$ & 1998 \\
\hline Jaén & Plan de expurgo del fondo bibliográfico & 2005 \\
\hline La Laguna & Manual de adquisiciones & 2009 \\
\hline La Laguna & Reglamento de expurgo & 2002 \\
\hline Las Palmas de Gran Canaria & Cómo donar libros a la Biblioteca & --- \\
\hline Lleida & Pautas de aceptación de donaciones y protocolo para los fondos especiales & 2011 \\
\hline Lleida & Pautas de Baja de documentos & 2011 \\
\hline Lleida & Pautas para el expurgo del fondo documental & 2011 \\
\hline Navarra & Orientaciones y criterios para donaciones & --- \\
\hline Pablo Olavide & Instrucciones para la suscripción y adquisición de documentos & 2010 \\
\hline Politécnica de Cartagena & Normativas de adquisición de material bibliográfico & --- \\
\hline Politécnica de Cartagena & Normativa de uso de suscripción de nuevos títulos de bases de datos & 2004 \\
\hline Politécnica de Cartagena & Normativa de uso de suscripción de nuevos títulos de publicaciones periódicas & 2002 \\
\hline Pompeu Fabra & Criterios para la aceptación de donaciones & --- \\
\hline Pontificia de Comillas & $\begin{array}{l}\text { Normativa para el mantenimiento del patrimonio bibliográfico y documental y } \\
\text { para la adquisición de documentos }\end{array}$ & 2002 \\
\hline Rey Juan Carlos & Normativa de donación, canje y depósito & 2010 \\
\hline Rey Juan Carlos & Normativa de expurgo & 2010 \\
\hline Rovira i Virgili & Política de aceptación de donaciones & 2008 \\
\hline UNED & Normas sobre donativos a la Biblioteca & --- \\
\hline Valladolid & Normativa para la aceptación de donaciones de material bibliográfico & 2011 \\
\hline Valladolid & Normativa de expurgo & 2006 \\
\hline Vigo & Criterios de selección de recursos de información & --- \\
\hline
\end{tabular}


listado y con elementos parecidos. El resto (BarceIona, Complutense, Vigo) se alejan de este modelo con explicaciones más extensas.

Los criterios según la frecuencia de aparición son:

- Lengua de la publicación: 8 documentos.

- Previsión de uso: 8 documentos.

- Contenido (temático de valor): 8 documentos.

- Adecuación a la colección: 7 documentos.

- Actualización (o fecha de publicación): 7 documentos.

- Prestigio (del autor y/o del editor): 6 documentos.

- Formatos (o soporte): 6 documentos.

- Calidad: 6 documentos.

- Duplicados (evitarlos): 5 documentos.

- Materia (o cobertura temática): 4 documentos.

- Soporte (apoyo a la docencia, al aprendizaje, a la investigación, a las tareas del personal...): 3 documentos

- Aspectos económicos: precio (2 documentos), disponibilidad de presupuesto (3 documentos), coste de procesamiento o mantenimiento (1 documento).

- Relevancia: 2 documentos.

- Completar una colección: 2 documentos.

- Encuadernación: 2 documentos.

En los 5 documentos que recogen criterios específicos (tabla III) se establecen dos distinciones:

a) Los textos de Alcalá y Vigo sólo presentan criterios para uno o más tipos de materiales (Alcalá: recursos electrónicos; Vigo: revistas y bases de datos).

b) Los 3 textos restantes mencionan las tipologías documentales en función de los tipos de usuarios miembros de la comunidad universitaria: alumnos (distinguiendo según los distintos niveles) y profesores, aludiendo a la docencia, aprendizaje e investigación. Estos criterios se completan con otros: obras de referencia (3 documentos); prensa ( 2 documentos); colecciones especiales (1 documento).

\subsubsection{Responsabilidad de la selección}

Este contenido se expone de manera semejante en todos los documentos a excepción del texto de la Universidad Autónoma de Barcelona, que lo organiza en función de la fuente de financiación y presupuesto.
Los documentos inician la exposición expresando que se trata de una actividad compartida, donde juegan un papel preeminente el personal docente e investigador y los bibliotecarios. A partir de aquí cada documento cuenta con sus propios matices:

- La Biblioteca de la Complutense o la de Murcia subrayan que es una actividad cooperativa donde participa toda la comunidad universitaria en mayor o menor grado.

- La Biblioteca de La Rioja precisa que el personal docente e investigador seleccionará directamente los fondos bibliográficos orientados a la investigación o a la docencia con cargo a cualquier presupuesto destinado a tal efecto y que los bibliotecarios de las secciones de Proceso e Información en sus respectivas áreas temáticas realizarán estudios de evaluación y observarán las necesidades de los usuarios. También, son los responsables de la actualización del fondo de bibliografía básica recomendada y de los fondos orientados a los alumnos con cargo al presupuesto de biblioteca. Precisa que se estudiarán las desideratas.

- La Biblioteca de la Carlos III establece que la responsabilidad es compartida entre los bibliotecarios de Proceso de Información Especializada (PIE), los coordinadores de biblioteca, y los docentes responsables de departamentos o áreas. El bibliotecario de PIE es responsable del desarrollo de sus áreas temáticas y se especializará en la investigación de recursos de información editorial, bibliográfica y datos de necesidades observadas que ayuden a los docentes en la selección.

- La Biblioteca de Sevilla matiza que el grado de participación de la comunidad universitaria en la selección depende del tipo de obra que se adquiera y de su finalidad.

Como se ha mencionado, la Biblioteca de la Autónoma de Barcelona plantea de otro modo las responsabilidades en la selección:

- Adquisiciones consorciadas: aprueba el Vicerrector de investigación a propuesta de la Dirección del Servicio de Bibliotecas.

- Suscripciones centralizadas: aprueba la Comisión de investigación a propuesta de los centros, departamentos y bibliotecas.

- Presupuestos de los centros: aprueba el decano, a propuesta del personal académico del centro y/o de la biblioteca.

- Subvenciones específicas de proyectos de investigación y de convenios: aprueba el responsable del proyecto a propuesta de los miembros del proyecto.

- Presupuesto distribuidos por el Vicerrectorado o los centros de las diferentes bibliotecas: 
Tabla III. Criterios específicos para la selección en los documentos sobre gestión de la colección de las bibliotecas universitarias españolas

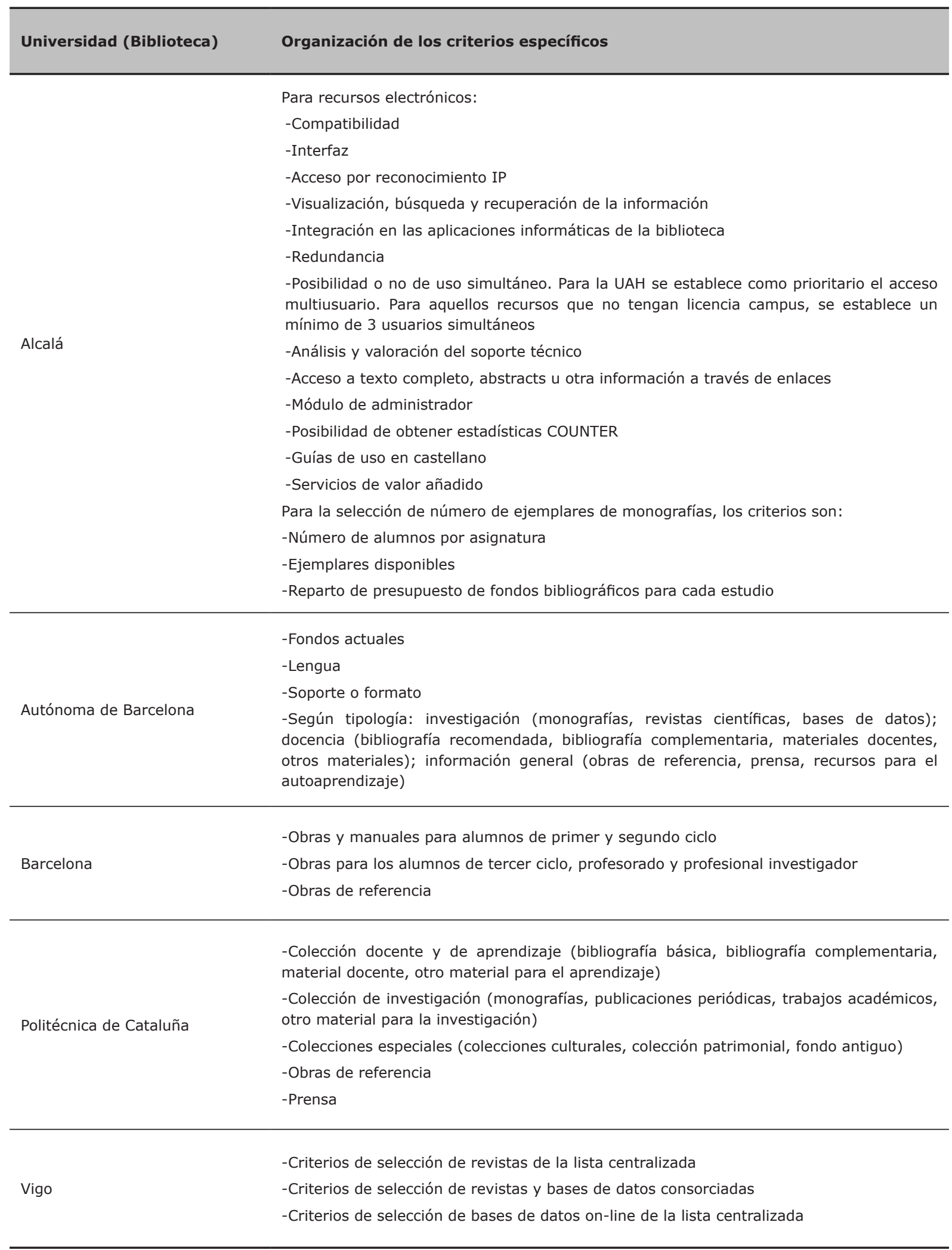


aprueba el jefe o coordinador de Biblioteca a propuesta de la biblioteca $y / 0$ del personal académico.

- Presupuesto específico ordinario para adquisiciones bibliográficas: aprueba el jefe y/o coordinador de biblioteca a propuesta de la biblioteca y/o del personal académico.

\subsubsection{Las fuentes}

La forma de presentar los apartados dedicados a las fuentes para la selección es muy parecida en los cuatro documentos que los incluyen y consiste en la elaboración de un listado con la tipología de las fuentes. La mayor coincidencia radica en las llamadas "fuentes básicas" donde se incluyen las recomendaciones de los docentes, las sugerencias de los alumnos, los programas académicos, etc.

Las clasificaciones para presentar los tipos de fuentes son las siguientes:

Biblioteca de la Universidad de Alcalá:

- Fuentes básicas:

- Bibliografía básica y complementaria recomendada por los profesores en cada asignatura para los diferentes estudios.

- Sugerencias de los alumnos.

- Proyectos de investigación.

- Informes de usos de libros prestados y reservados.

- Datos recogidos de inventario (deterioro, desaparición, etc. de ejemplares).

Fuentes complementarias: publicidad de editores, proveedores y libreros, repertorios especializados, libros a examen, índices de impacto.

Biblioteca de la Universidad de La Rioja:

- Catálogos de editores, libreros, etc.

- Información bibliográfica (elaborada por la biblioteca)

- Información de novedades: los nuevos servicios a través de Internet

- Obras a examen

- Información sobre recursos electrónicos

Biblioteca de la Universidad de Málaga

- Fuentes básicas (propuestas del PDI, programaciones académicas, desideratas de los usuarios).

Bibliografías (nacionales, comerciales, críticas, etc.).

- Otras fuentes útiles (catálogos: de libreros, de editores, etc.; libros a examen)
Biblioteca de la Universidad de Sevilla:

- Fuentes comerciales (catálogos: de libreros, de editores, de distribuidores, etc.).

- Fuentes bibliográficas (bibliografías nacionales, especializadas, catálogos de bibliotecas).

- Fuentes de carácter crítico (bibliografías críticas).

\section{CONCLUSIONES}

La planificación global de la gestión de la colección, plasmada en los documentos del tipo planes de gestión de la colección, es reducida, pues de las 71 bibliotecas universitarias españolas analizadas sólo el $14 \%$ cuentan con ellos (10 bibliotecas)

Las bibliotecas universitarias españolas sin ningún documento que regule la gestión de la colección al menos parcialmente ( 38 bibliotecas: $53,52 \%$ ) deben incorporar esta tarea entre sus objetivos de mejora. Para estas bibliotecas, los documentos que se han recopilado y analizado en este trabajo les pueden servir como fuente de consulta y conocimiento, pues, se debe tener presente que la gestión de la colección "es parte integral del planeamiento [...] sus metas y objetivos están integrados en él" (Aguado, 2011) y la planificación no puede faltar en el diseño y en la gestión de una unidad de información del siglo XXI.

El $32 \%$ de las bibliotecas universitarias españolas (23 bibliotecas) deben completar su política de gestión de la colección ampliándola para incorporar otros aspectos a los que ya tienen regulados y tender a la elaboración de planes de gestión de la colección completos. La ausencia de estos documentos se puede traducir en colecciones poco armónicas que incluyan desequilibrios, duplicados innecesarios, etc. En definitiva, que estén llevadas más por la improvisación que por la coherencia, situación que no se debe permitir nunca, pero aún menos en el contexto económico actual.

El estudio de los 10 planes de gestión de la colección refleja que estas bibliotecas universitarias españolas conciben la gestión de la colección como un conjunto de actividades que se deben hacer según unos criterios y directrices que permitan una coordinación. Y lo hacen desde un planteamiento amplio en el que se abarcan diferentes aspectos (selección, adquisición, evaluación, expurgo, presupuesto, proveedores, conservación y preservación, etc.) y en el que la selección y la adquisición siguen siendo los aspectos a los que se les dedica más atención, lo cual enlaza con la tradición de haber sido considerados en un principio los procesos centrales y prácticamente únicos, relacionados con la gestión de la colección.

De estos 10 planes se pueden considerar como más completos y que abordan con más detenimiento las cuestiones, los de las bibliotecas de las universidades de Murcia, Complutense de Ma- 
drid, Politécnica de Cataluña, Carlos III, La Rioja y Sevilla. Por ejemplo, todos se detienen en tratar los aspectos relacionados con los documentos electrónicos, cuestión fundamental en nuestros días.

Respecto a la selección, en el conjunto de documentos localizados, aparece formulada con un planteamiento parecido, muy identificada con los criterios de selección. En las normativas parciales se echa en falta una mayor referencia a los documentos electrónicos (a excepción de la Biblioteca de la Universidad de Alcalá) y a las iniciativas de acceso abierto.

La abundante información que aportan estos documentos hace aconsejable limitar este estudio a dar una visión de conjunto de la gestión de la colección y a centrarse en el proceso de la selección. En el futuro se ampliará el trabajo con estudios específicos dedicados al resto de aspectos, analizando los datos que aportan los documentos.

\section{NOTAS}

[1] Otros trabajos anteriores que abordan el tema de la selección y que nos parecen de interés son: H. Edelman (Selection..., 1979); B. Enright, et al. (Selection for survival: a review of acquisition and retention policies. London; The British Library, 1989); D. Spiller (Book selection: principies and practice. (5th ed) London; Library Association; Clive Bingley Book, 1992), S. H. Lee (Collection Development in a Digital Environment. New York; Haworth Press,1999); G. E. Evans (Developing Library and Information Center Colecction (4th ed.) Littleton; Libraries Unlimited, 2000); P. Johnson (Fundamentals..., 2004).

\section{BIBLIOGRAFÍA}

Aguado de Costa, A. (2011). Gestión de colecciones. Buenos Aires; Alfagrama ediciones, p.154.

ALA, Collection Management and Development Section (1996). Guide for written collection policy statements, a cargo de Joanne S. Anderson. ( $2^{\text {nd }}$ ed.) Chicago; American Library Association, p.36.

Evans, G. E. (1995). Developing Library and Information Center Collections. Englewood; CO, Libraries Unlimited (Library Science Text Series), p.556.

Fuentes Romero, J. J. (2010). La colección de materiales en las bibliotecas. Madrid; Arco/Libros, p.310.

Futas, E. (1995). Collection development policies and procedures. Phoenix; Oryx Press, p.349.

Gabriel, M. (1995). Collection Development and Collection Evaluation. Lanham; Md. \& London, Scarecrow Press, p.421.

Gardner, R. K. (1981). Library collections, their origin, selection, and development. New York; MacGrawHill, p.354.

Gómez Hernández, J. A. (1997). Biblioteconomía General y Aplicada: conceptos básicos de gestión de bibliotecas. Murcia; DM, p.302.

Gómez Hernández, J. A. (2002). Gestión de bibliotecas. Murcia; Universidad de Murcia, p.342.

IFLA, Sección de Adquisiciones y Desarrollo de las Colecciones (2001). Directrices para una política de desarrollo de las colecciones sobre la base del modelo Conspectus [en línea]. [Consulta: 30 junio 2012].

Disponible en: <http://archive.ifla.org/VII/s14/nd1/ gcdp-s.pdf $>$.

Solimine, G. (2010). La gestión de la colección. En: Solimine, G.; Domenico, G. di; Pérez Pulido, M. Gestión y planificación en bibliotecas. Buenos Aires; Alfagrama, p.320.

Whithe, G. W.; Crawford, G. A. (1997). Developing an information resources colection development policy. Collection Building, vol. 16 (2), 53-57. 
ANEXO I: Bibliotecas universitarias españolas con documentos sobre la gestión de la colección

\begin{tabular}{|c|c|c|c|}
\hline Universidad (Biblioteca) & $\begin{array}{l}\text { Planes de gestión } \\
\text { de la colección }\end{array}$ & $\begin{array}{l}\text { Normativas parciales sobre } \\
\text { gestión de la colección }\end{array}$ & $\begin{array}{c}\text { Observaciones de las } \\
\text { bibliotecas }\end{array}$ \\
\hline A Coruña & & & En proceso de redacción \\
\hline Abat Oliba CEU & & & $\begin{array}{l}\text { No disponen de normativas sobre } \\
\text { gestión de la colección. Siguen las } \\
\text { directrices que se contemplan en } \\
\text { el Reglamento de la biblioteca }\end{array}$ \\
\hline Alcalá & & X (selección) & $\begin{array}{l}\text { En elaboración la normativa sobre } \\
\text { adquisición }\end{array}$ \\
\hline Alfonso X El Sabio & $x$ & & \\
\hline Alicante & & $\begin{array}{l}\text { X (adquisiciones / suscripción } \\
\text { bases datos y pp. periódicas) }\end{array}$ & \\
\hline \multicolumn{4}{|l|}{ Almería } \\
\hline \multicolumn{4}{|l|}{ Antonio de Nebrija } \\
\hline Autónoma de Barcelona & $x$ & & $\begin{array}{l}\text { El actual está en revisión y será } \\
\text { sustituido en breve }\end{array}$ \\
\hline Autónoma de Madrid & & & $\begin{array}{l}\text { Dos grupos de mejora están } \\
\text { trabajando sobre } \\
\text { expurgo y libros electrónicos }\end{array}$ \\
\hline Barcelona & $x$ & & \\
\hline Burgos & & X (adquisiciones) & \\
\hline Cádiz & & X (expurgo y donación) & \\
\hline Camilo José Cela & & & $\begin{array}{l}\text { No disponen de normativas sobre } \\
\text { gestión de la colección }\end{array}$ \\
\hline Cantabria & & X (adquisiciones) & \\
\hline Carlos III de Madrid & $x$ & & \\
\hline Castilla La Mancha & & X (adquisiciones) & \\
\hline Católica de Ávila & & X (adquisiciones / expurgo) & \\
\hline \multicolumn{4}{|l|}{ Católica de San Antonio } \\
\hline \multicolumn{4}{|l|}{ Católica de Valencia } \\
\hline \multicolumn{4}{|l|}{ CEU Cardenal Herrera } \\
\hline Complutense de Madrid & $x$ & & \\
\hline
\end{tabular}

Han publicado el plan de

expurgo aplicado a la biblioteca

Córdoba

Maimónides, en el Boletín de

la Asociación Andaluza de

Bibliotecarios ( $n^{\circ} 112,2011$ )

Deusto

Europea de Madrid

No disponen de normativas sobre

gestión de la colección

Europea Miguel de Cervantes

$\begin{array}{ll}\text { Extremadura } & X \text { (donación / adquisiciones) } \begin{array}{l}\text { El documento sobre adquisiciones } \\ \text { es de uso interno al que nos han } \\ \text { facilitado la consulta }\end{array}\end{array}$

Francisco de Vitoria

No disponen de normativas sobre gestión de la colección

\begin{tabular}{ll}
\hline Girona \\
\hline Granada \\
\hline Huelva X (adquisiciones) \\
\hline IE Universidad
\end{tabular}




\begin{tabular}{|c|c|c|c|}
\hline \multicolumn{4}{|l|}{ Internacional de Catalunya } \\
\hline \multicolumn{4}{|l|}{ Internacional de la Rioja } \\
\hline Jaén & & $X($ expurgo) & $\begin{array}{l}\text { Programa de gestión de la } \\
\text { colección en proceso de elaboración }\end{array}$ \\
\hline \multicolumn{4}{|l|}{ Jaume I } \\
\hline La Laguna & & X (adquisiciones / expurgo) & \\
\hline La Rioja & $x$ & & \\
\hline Las Palmas de Gran Canaria & & X (donaciones) & $\begin{array}{l}\text { El plan de gestión de la colección } \\
\text { lo tienen como proyecto }\end{array}$ \\
\hline \multicolumn{4}{|l|}{ León } \\
\hline \multicolumn{4}{|l|}{ Les Illes Balears } \\
\hline Lleida & & $\begin{array}{l}\text { X (expurgo / donaciones / } \\
\text { baja ) }\end{array}$ & $\begin{array}{l}\text { Documentos de uso interno a los } \\
\text { que nos han facilitado la consulta }\end{array}$ \\
\hline Málaga & $x$ & & \\
\hline Miguel Hernández & & & En proceso de elaboración \\
\hline \multicolumn{4}{|l|}{ Mondragón } \\
\hline Murcia & $\mathrm{X}$ & & $\begin{array}{l}\text { Documentos de uso interno al que } \\
\text { nos han facilitado la consulta }\end{array}$ \\
\hline Navarra & & X (donaciones) & \\
\hline \multicolumn{4}{|l|}{ Oviedo } \\
\hline Pablo de Olavide & & X (adquisiciones) & \\
\hline País Vasco & & & En proceso de elaboración \\
\hline Politécnica de Cartagena & & $\begin{array}{c}\text { X (adquisiciones material } \\
\text { bibliográfico/suscripción bases } \\
\text { datos/suscripción pp. periódicas) }\end{array}$ & \\
\hline Politécnica de Catalunya & $x$ & & \\
\hline Politécnica de Madrid & & & No tienen ningún documento oficial \\
\hline Politécnica de Valencia & & & En proceso de elaboración \\
\hline Pompeu Fabra & & X (donaciones) & \\
\hline Pontificia de Comillas & & $\begin{array}{l}\mathrm{X} \text { (adquisiciones } \mathrm{y} \\
\text { mantenimiento) }\end{array}$ & \\
\hline Pontificia de Salamanca & & & $\begin{array}{l}\text { Manuales de procedimiento en } \\
\text { proceso de elaboración }\end{array}$ \\
\hline \multicolumn{4}{|l|}{ Pública de Navarra } \\
\hline \multicolumn{4}{|l|}{ Ramon Llull } \\
\hline Rey Juan Carlos & & $\begin{array}{l}\text { X (expurgo / donación, canje y } \\
\text { depósito) }\end{array}$ & \\
\hline Rovira i Virgili & & X (donaciones) & \\
\hline \multicolumn{4}{|l|}{ Salamanca } \\
\hline \multicolumn{4}{|l|}{ San Jorge } \\
\hline \multicolumn{4}{|l|}{ San Pablo-CEU } \\
\hline \multicolumn{4}{|l|}{ Santiago de Compostela } \\
\hline Sevilla & $x$ & & \\
\hline UNED & & X (donaciones) & \\
\hline \multicolumn{4}{|l|}{ Valencia } \\
\hline Valladolid & & X (donaciones / expurgo) & \\
\hline \multicolumn{4}{|l|}{ Vic } \\
\hline Vigo & & X (selección) & $\begin{array}{l}\text { Documentos de uso interno a los } \\
\text { que nos han facilitado la consulta }\end{array}$ \\
\hline Zaragoza & & & \\
\hline
\end{tabular}




\section{ANEXO II: Documentos sobre la gestión de la colección de las bibliotecas universitarias españolas}

\section{Planes de gestión de la colección}

-Universidad Alfonso X El Sabio (Biblioteca). Política de selección, adquisición y descarte [consulta: 3 mayo 2012]. Disponible en: <http://www.uax.es/ci/biblioteca.php/biblioteca\# >

-Universidad Autónoma de Barcelona (Biblioteca) (2005). Plan General de la colección. [consulta: 13 abril 2012]. Disponible en: <http://www.uab.cat/servlet/Satellite/fondo-y-equipamiento/planes-de-coleccion-1096482169488.html>

-Universidad Carlos III de Madrid (Biblioteca) (2008). Programa general de gestión de la colección [consulta: 3 mayo 2012]. Disponible en: <http://www.uc3m.es/portal/page/portal/biblioteca/sobre_la_biblioteca/colecciones/programa_ gestion_coleccion>

-Universidad Complutense de Madrid (Biblioteca) (2009). Política de gestión de la colecciones de la Biblioteca Complutense [consulta: 25 abril 2012]. Disponible en: <http://www.ucm.es/BUCM/intranet/29953.php>

-Universidad de Barcelona (Biblioteca) (2006). Criterios para la gestión de la colección en las bibliotecas de la Universidad de Barcelona [consulta: 4 mayo 2012]. Disponible en: <http://www.bib.ub.edu/fileadmin/arxius/gestiocolbibub.pdf>

-Universidad de La Rioja (Biblioteca). Plan de gestión de la colección [consulta: 4 mayo 2012]. Disponible en:

<http://biblioteca.unirioja.es/documentos/plan_gestion_coleccion.pdf>

-Universidad de Málaga (Biblioteca) (2007). Plan de gestión y desarrollo colección [consulta: 3 abril 2012]. Disponible en: <http://www.uma.es/publicadores/biblioteca/wwwuma/PlanGestionDesarrolloColeccion.pdf>

-Universidad de Sevilla (Biblioteca) (2006). Programa general de gestión y desarrollo de colecciones [consulta: 3 abril 2012]. Disponible en: <http://bib.us.es/servicios/compras_y_suscripciones/programa_formacion_coleccion/common/ Programa_Gestion_Coleccion.pdf>

-Universidad Politécnica de Cataluña (Biblioteca) (2009). Plan de gestión y desarrollo de la colección [consulta: 1 abril 2012]. Disponible en: <http://bibliotecnica.upc.edu/pla_colleccions_sbd_2009>

\section{Normativas y documentos parciales}

-Universidad Católica de Ávila (Biblioteca). Normativa de adquisiciones [consulta: 3 abril 2012]. Disponible en: <https:// www.ucavila.es/biblioteca/files/NORMATIVA\%20DE\%20ADQUISICIONES.pdf>

-Universidad Católica de Ávila (Biblioteca). Normativa de expurgo [consulta: 1 marzo 2012]. Disponible en: <https:// www.ucavila.es/biblioteca/files/NORMATIVA\%20DE\%20EXPURGO.pdf>

-Universidad de Alcalá de Henares (Biblioteca). Criterios para la selección de recursos de información [consulta: 1 marzo 2012]. Disponible en: <http://www.uah.es/biblioteca/documentos/Criterios\%20para\%20la\%20selecci\%C3\%B3n\%20 de\%20recursos\%20de\%20informaci\%C3\%B3n.pdf>

-Universidad de Alicante (Biblioteca). Normativa de adquisiciones de fondos bibliográficos [consulta: 12 marzo 2012 ]. Disponible en: <http://biblioteca.ua.es/es/documentos/docs/pdf/adquisiciones.pdf>

-Universidad de Alicante (Biblioteca). Normativa de suscripciones de publicaciones periódicas y bases de datos [consulta: 10 marzo 2012]. Disponible en: <http://biblioteca.ua.es/es/docs/normativas.html>

-Universidad de Burgos (Biblioteca). Instrucciones para la adquisición y el desarrollo de publicaciones [consulta: 5 marzo 2012]. Disponible en: <http://www.ubu.es/bubu/es/inforgeneralbubu/biblioteca/normativa/normativa-adquisiciones>

-Universidad de Cádiz (Biblioteca) (2010). Normativa de expurgo y donación [consulta: 21 marzo 2012]. Disponible en: <http://www.uca.es/area/biblioteca/sobrelabiblioteca/gestionyorganizacion/NormativaExpurgoyDonacion>

-Universidad de Cantabria (Biblioteca). Servicio de adquisiciones [consulta: 18 marzo 2012]. Disponible en: <http:// www.buc.unican.es/adqs>

-Universidad de Castilla-La Mancha (Biblioteca) (2004). Normativa del servicio de adquisiciones [consulta: 1 marzo 2012]. Disponible en: <http://biblioteca.uclm.es/normativa.html>

-Universidad de Extremadura (Biblioteca) (2007). Normativa de admisión de obras por donación [consulta: 8 marzo 2012]. Disponible en: <http://biblioteca.unex.es/informacion-general/normativa.html>

-Universidad de Extremadura (Biblioteca) (2010). Manual de procedimiento para las adquisiciones bibliográficas: monografías (documento de uso interno)

-Universidad de Huelva (Biblioteca) (1998). Normas para la adquisición de material bibliográfico y suscripción a publicaciones periódicas y bases de datos [consulta: 13 marzo 2012]. Disponible en: <http://www.uhu.es/biblioteca/normativa/ norma1.pdf> 
-Universidad de Jaén (Biblioteca) (2005). Plan de expurgo del fondo bibliográfico [consulta: 1 marzo 2012]. Disponible en: <https://www10.ujaen.es/sites/default/files/users/biblio/normativas/normas_04_plan_de_expurgo_2005.pdf>

-Universidad de La Laguna (Biblioteca) (2009). Manual de adquisiciones [consulta: 9 marzo 2012]. Disponible en: <http://www.bbtk.ull.es/Private/folder/institucional/bbtk/ftp/Informacion/manuales/Manual_Adquisiciones.pdf>

-Universidad de La Laguna (Biblioteca) (2002). Reglamento de expurgo [consulta: 1 marzo 2012]. Disponible en: <http:// www.bbtk.ull.es/Private/folder/institucional/bbtk/ftp/Informacion/documentacion/Reglamento_Expurgo.pdf>

-Universidad de Las Palmas de Gran Canaria (Biblioteca). Cómo donar libros a la biblioteca. [consulta: 3 mayo 2012]. Disponible en: <http://biblioteca.ulpgc.es/conocenos_donar>

-Universidad de Lleida (Biblioteca) (2011). Pautas de aceptación de donaciones y protocolo para los fondos especiales (documento de uso interno).

-Universidad de Lleida (Biblioteca) (2011). Pautas de Baja de documentos (documento de uso interno).

-Universidad de Lleida (Biblioteca) (2011). Pautas para el expurgo del fondo documental (documento de uso interno).

-Universidad de Murcia (Biblioteca) (2007). Procedimiento para la gestión de la colección (documento interno)

-Universidad de Valladolid (Biblioteca) (2006). Normativa de expurgo [consulta: 1 marzo 2012]. Disponible en: <http:// www.uva.es/export/sites/default/portal/adjuntos/documentos/1316075486487_expurgo1174378669729_expurgo.pdf>

-Universidad de Valladolid (Biblioteca) (2011). Normativa para la aceptación de donaciones de material bibliográfico [consulta: 19 marzo 2012]. Disponible en: <http://www.uva.es/export/sites/default/portal/adjuntos/documentos/1316074122573_normativa_donaciones_2011.pdf>

-Universidad de Vigo (Biblioteca). Criterios de selección de recursos de información (documento de uso interno).

-Universidad Nacional de Educación a Distancia. UNED (Biblioteca). Normas sobre donativos a la Biblioteca [consulta: 17 marzo 2012]. Disponible en: <http://portal.uned.es/pls/portal/docs/PAGE/UNED_MAIN/BIBLIOTECA/NORMATIVA/NORMASDONATIVOS.PDF>

-Universidad de Navarra (Biblioteca). Orientaciones y criterios para donaciones [consulta: 1 marzo 2012]. Disponible en: <http://www.unav.edu/web/biblioteca/donaciones >

-Universidad Pablo Olavide (Biblioteca) (2010). Instrucciones para la suscripción y adquisición de documentos [consulta: 1 marzo 2012]. Disponible en: <http://www.upo.es/biblioteca/normativa/de la bib/instrucciones suscripcion adquisicion_documentos/>

-Universidad Politécnica de Cartagena (Biblioteca). Normativas de adquisición de material bibliográfico [consulta: 1 marzo 2012]. Disponible en: <http://calamus.bib.upct.es/webdoc/servicios/adquisiciones_suscripciones/normativa_adquisiciones.pdf>

-Universidad Politécnica de Cartagena (Biblioteca) (2004). Normativa de uso de suscripción de nuevos títulos de bases de datos [consulta: 1 marzo 2012]. Disponible en: <http://calamus.bib.upct.es/webdoc/servicios/adquisiciones_suscripciones/normativa_bd.pdf

-Universidad Politécnica de Cartagena (Biblioteca) (2002). Normativa de uso de suscripción de nuevos títulos de publicaciones periódicas [consulta: 1 marzo 2012]. Disponible en: < http://calamus.bib.upct.es/webdoc/servicios/adquisiciones_suscripciones/normativa_pp.pdf

-Universidad Pompeu Fabra (Biblioteca). Criterios para la aceptación de donaciones [consulta: 1 marzo 2012]. Disponible en: <http://www.upf.edu/bibtic/es/coneixer/donacio.html>

-Universidad Pontificia de Comillas (Biblioteca) (2002). Normativa para el mantenimiento del patrimonio bibliográfico y documental y para la adquisición de documentos [consulta: 1 marzo 2012]. Disponible en: <http://www.upcomillas.es/ servicios/documentos/serv_bib_Norm_Adqui.pdf>

-Universidad Rey Juan Carlos (Biblioteca) (2010). Normativa de donación, canje y depósito. [consulta: 1 marzo 2012]. Disponible en: <http://www.urjc.es/biblioteca/Archivos/NORMATIVA\%20DE\%20DONACION\%20CANJE\%20Y\%20DEPOSITO.pdf>

-Universidad Rey Juan Carlos (Biblioteca) (2010). Normativa de expurgo [consulta: 1 marzo 2012]. Disponible en: <http://www.urjc.es/biblioteca/Archivos/NORMATIVA\%20EXPURGO.pdf>

-Universidad Rovira i Virgili (Biblioteca) (2008). Política de aceptación de donaciones [consulta: 1 marzo 2012]. Disponible en: <http://www.urv.cat/biblioteca/informacio general/donatius.html> 